\title{
Atendimento ao surdo na atenção básica: perspectiva da equipe multidisciplinar
}

\author{
Eliane Meira de Sousa ${ }^{I}$; Maria Antonieta Pereira Tigre Almeida ${ }^{2}$
}

\begin{abstract}
Resumo: A surdez é caracterizada pela perda da percepção normal dos sons. Os profissionais que atuam na atenção básica precisam estar preparados para acolher e prestar atendimento a toda a população, inclusive ao usuário com deficiência auditiva, pois esta deve considerar o indivíduo em sua particularidade, complexidade, integralidade e inserção sociocultural. A atenção básica é considerada, dentro da rede de atenção à saúde como o primeiro nível, sendo a porta de entrada do usuário para os serviços ofertados pelo sistema único de saúde, e tem o papel de desenvolver estratégias que possibilitem ao indivíduo viver de modo saudável, autônomo e sem limitações físicas ou de comunicação. Nesse sentido, o presente estudo teve como objetivo geral investigar o preparo da equipe multiprofissional quanto ao atendimento a pessoa surda na atenção básica, e como objetivos específicos: identificar as dificuldades encontradas pelos profissionais durante o atendimento ao surdo; evidenciar as estratégias de comunicação utilizadas pelos profissionais no atendimento ao surdo e verificar a existência de formação ou capacitação do profissional da atenção básica para atendimento ao surdo. O estudo foi realizado por meio de um levantamento bibliográfico através de busca eletrônica de trabalhos científicos. Foi possível perceber que o atendimento aos surdos na saúde pública está em processo de construção e ainda apresenta sérias deficiências. Assim, para sanar as dificuldades existentes é necessário a presença de acompanhante ou intérpretes preparados que estejam à disposição do serviço e a inclusão de curso de LIBRAS e de disciplinas que abordem os aspectos da comunicação com o surdo no currículo acadêmico das graduações da área de saúde.
\end{abstract}

Palavras-chave: Atenção primária a saúde, Deficiência auditiva, Libras, Comunicação.

\section{Attention to the deaf in basic care: perspective of the multidisciplinary team}

\begin{abstract}
Deafness is characterized by loss of normal perception of sounds. The professionals working in primary care need to be prepared to receive and provide care to the entire population, including the user with hearing impairment as this should consider the individual in its particularity, complexity, comprehensiveness and sociocultural integration. Primary care is considered, within the health care network as the first level, and the user's gateway to the services offered by the public health system, and has the role to develop strategies that enable the individual to live healthily, autonomous and without physical limitations or communication. In this sense, the present study aimed to investigate the preparation of the multi-professional team and service the deaf person in primary care, and as specific objectives: to identify the difficulties encountered by professionals in the service to the deaf; highlight the communication strategies used by professionals in serving the deaf and verify the existence of training or professional training of primary care to serve the deaf. The study was conducted through a literature through electronic search of scientific papers. It was possible to see that the service to the deaf public health is in the building process and still has serious shortcomings. So, to remedy the difficulties for a companion or prepared interpreters who are available to the service and the ongoing inclusion of LBS and disciplines that address aspects of communication with the deaf in the academic curriculum of health graduations is required .
\end{abstract}

Keywords: Primary health care, Hearing deficiency, Libras, Communication.

\footnotetext{
${ }^{1}$ Graduanda do $10^{\circ}$ semestre de enfermagem pela Faculdade Independente do Nordeste - FAINOR. ane.meira-@hotmail.com;

${ }^{2}$ Graduação em História pela Universidade Estadual do Sudoeste da Bahia. Pós - graduação em Psicopedagogia Clínica e Institucional pela Universidade Integrada Simonsen. Pós - Graduação em Educação Inclusiva pela Universidade Estadual da Bahia. Tem experiência na área de História, com ênfase em Interprete de Lingua de Sinais. Graduada em Letras Vernácula pela UESC no sistema UAB. É mestre em Linguística pela Universidade Estadual do Sudoeste da Bahia UESB. É Bacharel em Letras Libras Universidade Estadual de Santa Catarina (UFSC). Email: maptigre@gmail.com
} 
Id on Line Revista Multidisciplinar e de Psicologia

Id on Line Multidisciplinary and Psycology Journal

\section{Introdução}

A atenção básica é considerada, dentro da rede de atenção à saúde como o primeiro nível, sendo a porta de entrada do usuário para os serviços ofertados pelo sistema único de saúde (SUS). É caracterizada por um conjunto de ações de saúde, no âmbito individual e coletivo, que abrange "a promoção e a proteção da saúde, a prevenção de agravos, o diagnóstico, o tratamento, a reabilitação, a redução de danos e a manutenção da saúde" (BRASIL, 2012).

Neste contexto, os profissionais que atuam na atenção básica precisam estar preparados para acolher e prestar atendimento a toda a população, inclusive ao usuário com deficiência auditiva, pois, conforme Ohara e Saito (2010), a atenção básica deve considerar o indivíduo em sua particularidade, complexidade, integralidade e inserção sociocultural. Além disso, tem como papel desenvolver estratégias que possibilitem ao indivíduo viver de modo saudável, autônomo e sem limitações físicas ou de comunicação.

Em relação à comunicação, trata-se de um instrumento decisivo no diagnóstico, tratamento e prevenção, já que são utilizadas instruções verbais em vários procedimentos e, quando não podem ser compreendidas, levam ao comprometimento pela deficiência de captação das partes envolvidas (CHAVEIRO; BARBOSA; PORTO, 2008).

Quando os profissionais de saúde acolhem os usuários, torna-se necessário que se estabeleça um canal de comunicação que permita um entendimento de ambas as partes. $\mathrm{Na}$ maioria das vezes, essa comunicação se dá usando-se a linguagem verbal. Mas existem outras formas de comunicação, como a comunicação através da língua brasileira de sinais (LIBRAS), que é a língua oficial utilizada pela população surda brasileira, reconhecida como meio legal de comunicação e expressão (TEDESCO; JUNGES, 2013).

A surdez é caracterizada pela perda da percepção normal dos sons. De acordo com o aspecto da interferência na aquisição da linguagem e da fala, o déficit auditivo pode ser definido como perda média em decibéis, na zona conversacional (frequência de 500 - 1000 - 2000 hertz) para o melhor ouvido (BRASIL, 2006).

É grande a parcela de pessoas surdas na população brasileira. Segundo dados do censo demográfico de 2010 do Instituto Brasileiro de Geografia e Estatística (IBGE), cerca de 23,9\% da população do país apresenta pelo menos uma das deficiências investigadas, entre essas, a deficiência visual apresenta maior incidência, seguida da deficiência auditiva, declarada por $5,1 \%$ da população (IBGE, 2010). 
Diante disso, no que se concerne a assistência à saúde da pessoa com deficiência, as Unidades Básicas de Saúde e da Saúde da Família devem prestar assistência às pessoas com surdez como a qualquer cidadão (BRASIL, 2010). Isto significa que a assistência a pessoa com deficiência auditiva se insere no mesmo modelo assistencial geral utilizado pelos demais cidadãos, não devendo existir nenhum tipo de segregação ou diferenciação no atendimento que prejudique quaisquer das partes.

Assim, é de responsabilidade da atenção básica o desenvolvimento de práticas de cuidado à saúde direcionada para os indivíduos portadores de alguma deficiência, principalmente referente à circulação, participação social, inclusão e garantia dos seus direitos.

Considerando que a Atenção Básica é a porta de entrada para o Sistema Único de Saúde (SUS), torna-se necessário investigar se os profissionais de saúde estão preparados para acolher o surdo nas suas múltiplas necessidades, atendendo aos princípios da universalidade, integralidade e equidade.

Nesta perspectiva, o presente estudo teve como objetivo geral investigar o preparo da equipe multiprofissional quanto ao atendimento a pessoa surda na atenção básica, e como objetivos específicos: identificar as dificuldades encontradas pelos profissionais durante o atendimento ao surdo; evidenciar as estratégias de comunicação utilizadas pelos profissionais no atendimento ao surdo e verificar a existência de formação ou capacitação do profissional da atenção básica para atendimento ao surdo.

\section{Metodologia}

O estudo em questão constituiu-se de uma revisão da literatura especializada, por meio de artigos científicos.

Foi realizado um levantamento bibliográfico através de busca eletrônica de trabalhos científicos proclamados no: Scielo, Google Acadêmico, a partir de palavras - chaves.

Foram utilizados como critérios de inclusão para a realização deste estudo:

- Publicações do ano de 2006 ao ano de 2015;

- Veículo de publicação: artigos e dissertações;

- Idioma de publicação: português. 
E como critério de exclusão:

- Publicação de trabalhos sem caráter cientifico;

- Publicações antecedentes ao ano de 2006.

A pesquisa bibliográfica consiste em explicar e mostrar um problema, utilizando certos conhecimentos disponíveis a partir da temática em estudo. Incide ainda em revelar o pesquisador o conhecimento disponível na área, identificando as teorias produzidas, analisando-as e avaliando sua contribuição para auxiliar a compreender ou explicar o problema tendo a análise da investigação do texto (KOCHE, 1997).

\section{Resultados e Discussão}

No presente estudo foram analisados três artigos e duas dissertações como descritos no quadro 1.

Quadro 1. Trabalhos científicos sobre atendimento ao surdo na atenção básica

\begin{tabular}{|c|c|c|c|c|}
\hline Autor & $\begin{array}{c}\text { Ano de } \\
\text { publicação }\end{array}$ & Categoria & Revista & $\begin{array}{c}\text { Tipo de } \\
\text { pesquisa }\end{array}$ \\
\hline França & 2011 & $\begin{array}{c}\text { Dissertação } \\
\text { Universidade Federal } \\
\text { da Paraíba }\end{array}$ & $\begin{array}{c}\text { Estudo } \\
\text { quanti- } \\
\text { qualitativo }\end{array}$ \\
\hline Santos & 2015 & $\begin{array}{c}\text { Dissertação } \\
\text { Universidade Federal } \\
\text { do Rio Grande do } \\
\text { Norte }\end{array}$ & $\begin{array}{c}\text { Estudo } \\
\text { qualitativo } \\
\text { exploratório }\end{array}$ \\
\hline $\begin{array}{c}\text { Tedesco e } \\
\text { Junges }\end{array}$ & 2013 & Artigo & $\begin{array}{c}\text { Cad. De Saúde } \\
\text { Pública }\end{array}$ & $\begin{array}{c}\text { Estudo } \\
\text { qualitativo }\end{array}$ \\
\hline $\begin{array}{c}\text { Carvalho Filha, } \\
\text { Silva e Lando }\end{array}$ & 2015 & Artigo & $\begin{array}{c}\text { Revista Ciência \& } \\
\text { Saberes }\end{array}$ & $\begin{array}{c}\text { Estudo } \\
\text { qualitativo }\end{array}$ \\
\hline $\begin{array}{c}\text { Abreu, Freitas } \\
\text { e Rocha }\end{array}$ & 2015 & Artigo & $\begin{array}{c}\text { Brazilian Journal of } \\
\text { Surgery and Clinical } \\
\text { Research }\end{array}$ & $\begin{array}{c}\text { Estudo } \\
\text { qualitativo }\end{array}$ \\
\hline
\end{tabular}

Fonte: dados da pesquisa

Conforme o quadro 1 foi mais frequente o estudo de abordagem qualitativa. Segundo Nogueira-Martins e Bógus (2004) a abordagem qualitativa refere-se a estudos de significados, significações, ressignificações, representações psíquicas, representações sociais, 
Id on Line Revista Multidisciplinar e de Psicologia

Id on Line Multidisciplinary and Psycology Journal

simbolizações, simbolismos, percepções, pontos de vista, perspectivas, vivências, experiências de vida, analogias. A abordagem qualitativa é aplicada por se adequar à investigação das percepções e das opiniões que as pessoas emitem a respeito de como vivem, sentem, pensam, constroem a si mesmas e seus artefatos (MINAYO, 2006).

Após a leitura minuciosa das publicações é possível observar que todos os autores destacam os diversos problemas relacionados à comunicação efetiva no atendimento em saúde de indivíduos surdos.

Em pesquisa realizada por França (2011) com 156 profissionais da saúde atuantes nas UESF e nos Núcleos de apoio à Saúde da Família, em Campina Grande-PB, com o objetivo de identificar as dificuldades enfrentadas por estes na atenção primária durante o atendimento ao surdo, o autor constatou que a maior parte das respostas foi em relação às dificuldades de comunicação. Os profissionais alegaram que o não entendimento do que o surdo quer dizer impossibilita o conhecimento da real necessidade do usuário, e isso compromete a prescrição e orientações sobre os cuidados em saúde. A alternativa encontrada pelos profissionais foi a solicitação da presença de um familiar ou pessoa do convívio diário do usuário para intermediar a comunicação.

Além da dificuldade de comunicação citada pelos profissionais na pesquisa acima referida, foi mencionada também a dificuldade de transmitir as informações de forma que o surdo possa compreender a intervenção profissional. França (2011) ressalta que alguns profissionais alegaram ter o mínimo de conhecimento em linguagem não verbal necessária para compreender as necessidades de saúde do usuário, sendo a maior dificuldade a de fazer o surdo compreender as condutas terapêuticas.

Corroborando com a pesquisa dos autores supracitados, Tedesco e Junges (2013) também destacaram a dificuldade dos profissionais na comunicação durante o atendimento. No estudo, cujo objetivo era apontar os desafios que os profissionais de saúde comunitária vivenciam no atendimento aos usuários surdos, os profissionais foram questionados sobre a realização do atendimento a pessoas surdas no dia a dia das unidades. Os entrevistados relataram como ferramentas utilizadas a comunicação escrita, a presença de um familiar ou acompanhante e o uso de gestos. Contudo, os autores enfatizam que geralmente os surdos utilizam gestos e expressões que não pertencem a língua brasileira de sinais (LIBRAS), mas que são entendidas universalmente como expressões de dor ou apontar o local para auxiliar o 
Id on Line Revista Multidisciplinar e de Psicologia

Id on Line Multidisciplinary and Psycology Journal

atendimento. Evidenciam ainda que os profissionais sentiam-se desconfortáveis ao atender as pessoas surdas, pois se sentiam despreparados para esse tipo de atendimento.

Nesse mesmo sentido, Santos (2015) entrevistou 21 profissionais de saúde que atuam em uma Unidade Básica de Saúde na cidade de Natal-RN visando identificar suas percepções frente ao atendimento de pacientes surdos. Corroborando com as pesquisas referidas acima, verificou-se no estudo que $100 \%$ dos profissionais referiram ter dificuldade no atendimento ao surdo por não se sentirem capacitados para atendê-los. Também foi questionado quanto ao conhecimento da lei 10.436, de 24 de abril de 2002, que reconhece a LIBRAS como língua oficial no Brasil e $81 \%$ dos profissionais disseram não conhecer a citada lei. $\mathrm{O}$ autor aponta como solução a utilização de um intérprete capacitado ou a capacitação do profissional da saúde.

Concordando com o autor supracitado, Santos e Shiratori (2004) ao pontuar como a principal dificuldade enfrentada pelos usuários surdos a comunicação, citam como proposta o auxílio de um intérprete em LIBRAS ou profissionais que saibam se comunicar por esta língua.

A língua brasileira de sinais (LIBRAS) é a segunda língua oficial do Brasil, legalizada pela Lei Federal $n^{\circ}$ 10.436/02 e regulamentada pelo Decreto $n^{\circ} 5.626$ de 22 de dezembro de 2005 e, desde então, tem sido divulgada nos mais variados setores sociais, educacionais e de saúde, embora ainda desconhecida em várias instâncias (BRASIL, 2002, 2005).

Apesar disso, sabe-se que a maioria dos profissionais não conhece a LIBRAS e nos serviços de saúde não são disponibilizados intérpretes. Na maioria das vezes o acompanhante é quem explica ao profissional os problemas de saúde apresentados pelo surdo, o que acaba afetando a sua cidadania, por depender de outras pessoas para ter acesso às informações que poderiam melhorar a sua qualidade de vida (SÁ, 2006).

Assim, torna-se imprescindível considerar que os profissionais de saúde precisam ser capazes de atender os pacientes surdos. Neste sentido, Sá (2006) ressalta que é inconcebível que a maioria dos serviços de saúde ainda não ofereça aos surdos profissionais capacitados para se comunicar devidamente por meio de LIBRAS.

França (2011) questionou os profissionais participantes da pesquisa quanto ao conhecimento ou se já ouviram falar em LIBRAS, e constatou que grande parte $(78,2 \%)$ dos participantes afirmou que sim. Contudo, quando foram interrogados se utilizavam a comunicação por meio de LIBRAS, praticamente todos $(98,1 \%)$ os profissionais responderam 
Id on Line Revista Multidisciplinar e de Psicologia

Id on Line Multidisciplinary and Psycology Journal

não. $\mathrm{O}$ decreto $\mathrm{n}^{\circ} 5.626$ garante a presença nos serviços de saúde de profissionais capacitados para uso de LIBRAS ou para a tradução e interpretação, o que quase nunca acontece. Na pesquisa também foi discutido se os profissionais haviam recebido algum tipo de instrução na graduação para se comunicar com o surdo, e 98,7\% responderam não. Para o autor, isso demonstra a necessidade de maior ênfase no ensino de estratégias de comunicação com o surdo nos cursos de graduação da área de saúde.

Contribuindo com essa questão, Souza e Porrozzi (2009) recomenda que os profissionais de saúde tenham, nos currículos de seus respectivos cursos, incluída a disciplina de Libras, não como eletiva, mas sim como crédito obrigatório. Para eles, essa medida proporcionaria a aquisição de saberes que iriam modificar as atitudes destes profissionais em relação ao atendimento prestado aos clientes surdos, aos seus familiares, assim como também uma maior interação em situações de convívio profissional com colegas surdos, contribuindo para otimização da atuação do profissional, da atenção à saúde e do ato de cuidar.

No estudo de Santos (2015), além da visão dos profissionais, este buscou conhecer a percepção do usuário surdo quanto ao atendimento, e contou com o depoimento de 8 participantes surdos. Os relatos deixaram transparecer que estes não são bem atendidos devido a dificuldade de comunicação. $\mathrm{O}$ autor ressalta ainda que os pacientes surdos buscam atendimento médico com menor frequência quando comparado a pacientes ouvintes, e esse fato se deve justamente a deficiência na comunicação, e que em alguns casos pode até não encontrar solução para seu problema de saúde.

Nesse mesmo enfoque, Carvalho Filha, Silva e Lando (2015) buscou avaliar o cuidado ao surdo na Atenção Primária à Saúde em Caxias-MA na percepção do próprio usuário. A pesquisa foi realizada com 250 indivíduos da Associação para Surdos que se reúnem semanalmente para discutir temáticas relacionadas às suas limitações e ganhos, bem como aprenderem e socializarem a LIBRAS. Do ponto de vista dos surdos, nota-se que a maior barreira para o atendimento relatada por estes também está relacionada à falta de comunicação eficiente. Os autores observam que se o profissional não dominar a LIBRAS dificilmente irá conseguir compreender a real necessidade do surdo e assim não poderá oferecer os cuidados que de fato o cliente necessita.

Ainda sobre essa temática, a pesquisa de Abreu, Freitas e Rocha (2015) analisou a percepção de 16 surdos em relação ao sistema de comunicação das Unidades de Atenção 
Id on Line Revista Multidisciplinar e de Psicologia

Id on Line Multidisciplinary and Psycology Journal

Primária à Saúde do município de Varginha-MG. Em relação ao sentimento do surdo diante do atendimento prestado pelos profissionais de saúde, verificou-se que $50 \%$ dos entrevistados afirmaram que se sentem desprezados e com medo. Mais uma vez, o maior obstáculo citado no atendimento foi a comunicação com os profissionais, além disso, citaram também a falta de conhecimento de LIBRAS, paciência por parte dos profissionais e a falta de intérpretes no local de atendimento.

É possível perceber que os trabalhos analisados estão de acordo nas propostas para melhoria do atendimento aos surdos na atenção básica. Ao final do trabalho de França (2011), foi solicitado aos profissionais sugestões para a melhoria da comunicação com o surdo no atendimento na atenção básica e foi citada a capacitação voltada para esse tipo de atendimento; a presença de acompanhante ou intérpretes preparados à disposição do serviço e a inclusão de curso de LIBRAS e de disciplinas que abordem os aspectos da comunicação com o surdo no currículo acadêmico das graduações da área de saúde.

Confirmando as sugestões citadas anteriormente, Abreu, Freitas e Rocha (2015) também recomenda a inclusão de LIBRAS como disciplina obrigatória para os cursos da área da saúde e implantação de educação continuada voltada para LIBRAS com profissionais já atuantes.

É fundamental que o profissional de saúde esteja preparado para prestar um atendimento efetivo a esses indivíduos, de forma que a qualidade da assistência à saúde prestada não seja comprometida (FENEIS, 2008).

Cardoso, Rodrigues e Bachion (2006) afirmam a necessidade de atendimento especial pelos profissionais de saúde às pessoas surdas, uma vez que esses indivíduos possuem cultura e linguagem diferenciadas e essas particularidades precisam ser respeitadas. No contexto geral da assistência à saúde, os surdos se sentem discriminados, e inclusive, muitos relatam medo por não entenderem o que os profissionais proferem, evidenciando assim que precisam de atenção especial.

\section{Considerações Finais}

Nota-se então que os obstáculos encontrados pelas pessoas surdas na comunicação com os profissionais no atendimento na atenção primária são a falta de conhecimento e utilização de LIBRAS e a falta de intérpretes capacitados nos locais de atendimento. 
Id on Line Revista Multidisciplinar e de Psicologia

Id on Line Multidisciplinary and Psycology Journal

Cabe ao profissional que atua na atenção básica não só competência técnica, mas o conhecimento aprofundado e habilidades que favoreçam o estabelecimento de processos de comunicação e relações de caráter interpessoal, para que a troca de mensagens e informações seja de forma clara e eficiente. Pois ao procurar os serviços de saúde, as pessoas buscam além de acolhimento, relações solidárias e de confiança com os profissionais para resolução do seu problema de saúde, e para que esse vínculo seja criado é necessário que o processo de comunicação seja efetivo.

Com isso, ressalta-se a importância de enfatizar durante a graduação disciplinas que abordem os aspectos da comunicação com o surdo, a inclusão de LIBRAS como disciplina obrigatória nos cursos da área de saúde, e também a implantação de educação continuada de LIBRAS voltada para profissionais já atuantes.

\section{Referências}

ABREU, J. C.; FREITAS, J. M. R.; ROCHA, L. L. V. A percepção dos surdos em relação ao sistema de comunicação das unidades de atenção primária à saúde. Brazilian Journal of Surgery and Clinical Research, v. 9, n. 1, p. 06-11, 2015.

BRASIL. Ministério da Saúde. Lei $n^{o}$ 10.436, de 24 de abril de 2002. Dispões sobra a Língua Brasileira de Sinais e dá outras providências. Brasília: Ministério da Saúde, 2002.

BRASIL. Decreto $n^{\circ} 5.626$, de 22 de dezembro de 2005. Regulamenta a Lei ${ }^{\circ} 10.436$ de 24 de abril de 2002 que dispõe sobre a Língua Brasileira de Sinais (LIBRAS), e o art 18 da lei no 10.098 de 19 de dezembro de 2000. Brasília-DF, 2005.

BRASIL. Ministério da Educação. Secretaria de Educação Especial. Educação infantil. Surdez. Saberes e práticas da inclusão. Dificuldade de comunicação e sinalização. BrasíliaDF, 2006.

BRASIL. Ministério da Saúde. Secretaria de Atenção à Saúde. Departamento de ações programáticas estratégicas. Atenção à saúde da pessoa com deficiência no Sistema Único de Saúde (SUS). 1. ed. 1. reimp. Brasília: Ministério da Saúde, 2010.

BRASIL. Ministério da Saúde. Política Nacional da Atenção Básica (PNAB). Brasília, DF: MS, 2012.

CARDOSO, A. H. A.; RODRIGUES, K. G.; BACHION, M. M. Percepção da pessoa com surdez severa e/ou profunda acerca do processo de comunicação durante seu atendimento de saúde. Rev. Latino-Am. Enfermagem, v. 14, n. 4, p. 553-560, 2006. 
Id on Line Revista Multidisciplinar e de Psicologia

Id on Line Multidisciplinary and Psycology Journal

CARVALHO FILHA, F. S. S.; SILVA, S. R.; LANDO, G. A. Cuidado ao surdo: conexões com o direito à saúde. Revista Ciência \& Saberes, v. 1, n. 1, p. 31-38, 2015.

CHAVEIRO, N.; BARBOSA, M. A.; PORTO, C. C. Revisão de literatura sobre o atendimento ao paciente surdo pelos profissionais de saúde. Rev. Esc. Enferm., USP, São Paulo, SP, v. 42, n. 3, p. 578-583, 2008.

FEDERAÇÃO NACIONAL DE EDUCAÇÃO E INTEGRAÇÃO DOS SURDOS (FENEIS). Libras: Língua Brasileira de Sinais, 2008.

FRANÇA, E. G. Atenção à saúde do surdo na perspectiva do profissional de saúde. 2011. 84f. Dissertação (Mestrado) - Universidade Estadual da Paraíba, Campina Grande, PB, 2011.

INSTITUTO BRASILEIRO DE GEOGRAFIA E ESTATÍSTICA (IBGE). Censo

Demográfico. 2010. Disponível em:

<ftp://ftp.ibge.gov.br/censos/censodemografico2010/caracteristicas_gerais_religiao_deficienc $\mathrm{ia} /$ caracteristicas_religiai_defiencias.pdf $>$. Acesso em: 02 set. 2016.

KÖCHE, J. C. Fundamentos de metodologia científica: teoria da ciência e prática da pesquisa. 14. Ed. Petrópolis: Vozes, 1997.

MINAYO, M. C. S. Pesquisa avaliativa por triangulação de métodos. In: BOSI, M. L. M.; MERCADO, F. J. Avaliação qualitativa de programas de saúde: enfoques emergentes. Petrópolis, RJ: Vozes, 2006. p. 163-90.

NOGUEIRA-MARTINS, M. C. F.; BÓGUS, C. M. Considerações sobre a metodologia qualitativa como recurso para o estudo das ações de humanização em saúde. Saúde e Sociedade, v. 13, n. 3, p. 44-57, 2004.

OHARA, E. C. C.; SAITO, R. X. S. Saúde da família: considerações teóricas e aplicabilidade. 2. ed. São Paulo: Martinari, 2010.

SÁ, N. R. L. Cultura, poder e educação dos surdos. São Paulo: Edua, 2006.

SANTOS, E. M.; SHIRATORI, K. As necessidades de saúde no mundo do silêncio: um diálogo com os surdos. Revista Eletrônica de Enfermagem, v. 6, n. 1, p. 68-76, 2004.

SANTOS, Paulo Roberto de Andrade. Percepção da equipe de saúde, discentes e usuários sobre a comunicação com indivíduos surdos na atenção primária. 2015. 51f. Dissertação (Mestrado) - Universidade Federal do Rio Grande do Norte, Natal, RN, 2015.

SOUZA, M. T.; POROZZI, R. Ensino de Libras para os profissionais de Saúde: uma necessidade premente. Revista Práxis, ano 1, n. 2, 2009.

TEDESCO, J. R.; JUNGES, J. R. Desafios da prática do acolhimento de surdos na atenção primária. Cad. Saúde Púb 


\section{Como citar este artigo (Formato ABNT):}

SOUZA, E.M.; ALMEIDA, M.A.P.T. Atendimento ao surdo na atenção básica: perspectiva da equipe multidisciplinar. Id on Line Revista Multidisciplinar e de Psicologia, Janeiro de 2017, vol.10, n.33, p. 72-82. ISSN: 1981-1179.

Recebido: $22 / 11 / 2016$

Aceito: $25 / 11 / 2016$ 